\title{
Quality of life, depression, and psychosocial mechanisms of suicide risk in prostate cancer
}

\author{
Dean A. Tripp ${ }^{1,2,3}$; Valentina Mihajlovic ${ }^{1}$; Katherine Fretz ${ }^{1}$; Gagan Fervaha ${ }^{3}$; Jason Izard $^{3,4}$; \\ Rebecca Corby ${ }^{1}$; D. Robert Siemens ${ }^{3}$ \\ ${ }^{1}$ Departments of Psychology, Queen's University, Kingston, ON, Canada; ${ }^{2}$ Department of Anesthesiology, Queen's \\ University, Kingston, ON, Canada; ${ }^{3}$ Department of Urology, Queen's University, Kingston, ON, Canada; \\ ${ }^{4}$ Department of Oncology, Queen's University, Kingston, ON, Canada
}

Funding: This research was supported by Ride for Dad/Prostate Cancer Fight Foundation

Cite as: Can Urol Assoc J 2020 May 12; Epub ahead of print. http://dx.doi.org/10.5489/cuaj.6310

Published online May 12, 2020

$* * *$

\section{Abstract}

Introduction: Prostate cancer (PCa) is the most common non-cutaneous cancer in men and is usually identified at a stage at which prolonged survival is expected. Therefore, strategies to address survivorship and promote well-being are crucial. This study's aim was to better understand suicidal behavior in $\mathrm{PCa}$ patients by examining psychosocial mediators (i.e., depression, psychache, perceived burdensomeness [PB], thwarted belongingness [TB]) in the relationship between quality of life (PCa-QoL) and suicide risk.

Methods: Four hundred and six men with PCa (Median age 69.35 years, standard deviation 7.79) completed an online survey on various psychosocial variables associated with suicide risk. A combined serial/parallel mediation model tested whether depression, in serial with both psychache and $\mathrm{PB} / \mathrm{TB}$, mediated the relationship between $\mathrm{PCa}-\mathrm{Q}$ oL and suicide risk.

Results: Over $14 \%$ of participants' self-reports indicated clinically significant suicide risk. Poorer PCa-QoL was related to greater depression, which was related to both greater psychache and $\mathrm{PB} / \mathrm{TB}$, which was associated with greater suicide risk. The serial mediation effect of depression and psychache was significantly stronger than that of depression and PB/TB. PCaQoL did not predict suicide risk through depression alone, showing that depressive symptoms affect suicide risk through psychache and PB/TB.

Conclusions: Given the alarming estimate of individuals at-risk for suicide in this study, clinicians should consider patients with poorer PCa-QoL and elevated depression for psychosocial referral or management. Psychache (i.e., psychological pain) and PB/TB (i.e., poor 
social fit) may be important targets for reducing suicide risk intervention beyond the impact of depression alone. 


\section{Introduction}

Prostate cancer $(\mathrm{PCa})$ is the most common male solid malignancy in North America. ${ }^{1}$ Indeed, an estimated 35,720 PCa-associated deaths are expected to occur in Canada ${ }^{2}$ and the Unites States in $2019 .{ }^{3}$ In spite of these alarming numbers, among all cancers and for all stages combined, PCa carries the most favorable 5-year survival rate. ${ }^{1}$ With a large cohort living after their PCa diagnosis, attention on survivorship issues and quality of life (QoL) as measured by patientreported outcomes are important foci of ongoing research. ${ }^{4}$

PCa patients experience a host of intrapersonal and interpersonal difficulties, with $60 \%$ of patients reporting decision-related distress. ${ }^{5}$ Approximately $18 \%$ of patients report depression following PCa treatment, ${ }^{6}$ which is concerning given depression shares a close relationship with suicide. ${ }^{7}$ Population-based studies suggest that men with PCa are at increased risk of death by suicide. ${ }^{8-13}$ Despite depression and suicidal ideation sharing an association, $30 \%$ of suicides among PCa patients were completed by patients without a diagnosis of depression. ${ }^{14}$

Although depression is a predictor of suicidal behaviour, ${ }^{7}$ two prominent theories offer specific explanations for processes related to suicidal behaviour: psychache and the Interpersonal Theory of Suicide (IPTS). Psychache refers to intense psychological pain, and is an important variable leading to suicidal behaviour; suicide occurs when psychache is deemed unbearable. ${ }^{15-19}$ Conversely, the IPTS suggests that the experience of two constructs, perceived burdensomeness (PB; the belief that one's existence is a burden on others) and thwarted belongingness (TB; the experience that one feels socially isolated), contribute to an individual's suicidal ideation. ${ }^{20}$ Indeed, the interaction between PB and TB is strongly associated with suicidal ideation. ${ }^{21-22}$

Despite the explanatory abilities of both theories, neither has been directly examined in men with PCa. One objective of this study was to produce an updated estimate of suicide risk in a PCa cohort. Given the association between lower PCa-QoL and increased depression, ${ }^{23-24}$ the primary objective was to model the relationship between measurements of PCa-QoL, depression, suicide risk, and variables from these theories. It was hypothesized that lower PCa-QoL would be associated with greater depressive symptoms, and greater depressive symptoms with increased suicide risk through the experience of either psychache and the primary components of the IPTS (i.e., PB/TB). Both theories of suicide were suggested as significant mediators of suicide risk; however, due to the burden that many cancer patients express having on their loved ones, ${ }^{25}$ it was further hypothesized that the IPTS (i.e., PB/TB) would be a stronger mediator of suicide risk than psychache in individuals with PCa.

\section{Methods}

\section{Participants}

A sample of 406 adult males were recruited for this study. Participants were recruited internationally through online support groups by posting on their webpages or via email. Patients were also recruited from PCa clinics at the Kingston Health Sciences Centre. To be eligible to 
participate, individuals had to be: diagnosed with $\mathrm{PCa}, 18$ years of age or older, and fluent in English. Demographic information for participants is outlined in Table 1.

\section{[INSERT TABLE 1 HERE]}

\section{Procedure}

This study was approved by the Queen's University Health Sciences and Affiliated Teaching Hospitals Research Ethics Board. Potential participants opened the survey link, and were provided the letter of information before consenting to participate. Participants had the option of declining any questions they felt uncomfortable answering or discontuning the survey. Although the questions in this survey were of a sensitive nature, participation in online research assessing suicidal behaviour generally does not cause harmful distress to research participants. ${ }^{26} \mathrm{All}$ participants received debriefing information that included online mental health resources.

\section{Measures}

All measures used in this study have been found to be valid and reliable, as described in the measures' original papers. In the current study, the internal consistency was calculated and found to be acceptable or higher for all measures (Cronbach's $\alpha$ s $>0.66$ ).

\section{Prostate cancer-quality of life}

PCa-QoL was assessed using the Functional Assessment of Cancer Therapy - Prostate instrument (FACT-P). ${ }^{27-28}$ This measure asseses general QoL (i.e., physical, social/family, emotional, and functional well-being), and concerns related to PCa (i.e., sexuality, bowel/bladder functioning, pain). There are 27 general items and $12 \mathrm{PCa}$-specific items, rated on extent to which each statement applies to the individual in the past seven days on a scale from 0 (not at all) to 4 (very much). Higher scores indicate a higher QoL.

\section{Depression.}

Participant's depressive symptoms were assessed using the Patient Health Questionnaire-9 (PHQ-9) ${ }^{29}$ This measure is based on the DSM-IV criteria for major depression. Respondents rated the extent to which nine symptoms of depression have bothered them over the past two weeks on a scale from 0 (not at all) to 3 (nearly every day). Higher scores indicate elevated depressive symptoms.

\section{Psychache}

Psychache was assessed using the Psychache Scale. ${ }^{30}$ This measure assesses the extent to which respondent's experience psychache (i.e., general psychological pain) by asking them to rate how frequently each of the 13 items occur on a scale from 0 (never) to 5 (always). Higher scores are indicative of higher levels of psychache. 


\section{Perceived burdensomeness and thwarted belongingness}

$\mathrm{PB} / \mathrm{TB}$ was measured using the 10-item version of the Interpersonal Needs Questionnaire (INQ10). ${ }^{31}$ Respondents rate the extent to which they agree with each statement to reflect how they have been feeling recently on a scale from 1 (not at all true for me) to 7 (very true for me). Higher scores reflect higher levels of $\mathrm{PB} / \mathrm{TB}$.

\section{Suicide risk}

Risk of suicide was measured using the Suicide Behaviours Questionnaire Revised (SBQ-R). ${ }^{32}$ This measure includes four items that assess respondents' history of suicidal ideation and behaviors as well as their future risk of suicide. Higher scores indicate greater suicide risk. Scores $\geq 7$ indicate clinically significant suicide risk in an adult general population, whereas scores $\geq 8$ indicate clinically significant suicide risk in an adult psychiatric inpatient population. These cut-offs were described as maximizing sensitivity and specificity in predicting suicide-risk status in the original paper.

\section{Data preparation}

Prior to data analysis, data were inspected for any irregularities such as impossible and missing values. Due to a coding error in one iteration of the online survey, the INQ-10 measure for 159 participants had possible response values ranging from one to ten instead of one to seven. Therefore, those participants' responses were scaled to be on a one to seven scale. The following conversions from participants original scores to new scaled scores were made: $1=1,2=1.66,3$ $=2.33,4=3,5=3.66,6=4.33,7=5,8=5.66,9=6.33,10=7$. Finally, total scores from the INQ-10 that were scaled were rounded up or down to account for decimal values, making the values more comparable to the unscaled values. Analyes using the rounded and unrounded values yielded identical results; therefore, analyses using the rounded values are preseted in this paper. Total INQ-10 scores were compared across groups with and without the coding error and no significant differences were found, suggesting that the error did not seem to significantly impact the reliability of the scores. As such, the validity of the instrument was likely preserved.

Prior to total score calculations for all variables, missing data were imputed. Participants had to have completed at least $80 \%$ of a particular measure in order to calculate a total score. If $20 \%$ or less of their data were missing, values were imputed by using the mean of their other responses in place of those missing values according to Tabachnick and Fidell's data management procedures. ${ }^{33}$ Next, total scores were calculated by summing up participant's responses in each measure.

\section{Data analysis}

Frequency analyses were used to calculate an estimate of the percentage of participants who were at risk for suicide compared to the general adult and inpatient population cut-offs. To test the hypothesis that depressive symptoms in serial with psychache, as well as depressive symptoms in serial with $\mathrm{PB} / \mathrm{TB}$, would mediate the relationship between $\mathrm{PCa}-\mathrm{Q}$ oL and suicide 
risk, model 81 from Hayes' PROCESS Macro for SPSS was used. ${ }^{34}$ This model combines both parallel and serial mediation effects, which allows one to examine the process through which a predictor variable leads to an outcome variable through a mediator variable which travels through two additional mediation paths in parallel. As such, the two suicide theories could be directly compared by examining the effect of $\mathrm{PCa}-\mathrm{QoL}$ on suicide risk through its effect on depression, and through depression's effects on each of the theory's variables, psychache and $\mathrm{PB} / \mathrm{TB}$, separately. Simply put, this model examines two chains of events leading from a predictor to an outcome. $\mathrm{PCa}-\mathrm{QoL}$ was entered into the model as the predictor variable, depression was entered as a mediator, psychache and $\mathrm{PB} / \mathrm{TB}$ were entered as two parallel mediators in serial with depression, and suicide risk was entered as the outcome variable. The model was specified to create 10,000 bias-corrected bootstrap samples of data upon which to evaluate the indirect (i.e., mediation) effects. Bootstrapping is a powerful non-parametric statistical technique that does not rely on distributional assumptions given that sampling distributions are generated by drawing from one's own data with replacement. Mediation effects were deemed statistically significant if the $95 \%$ confidence interval (CI) for an effect was entirely above or below zero. All effects described for the mediation model represent unstandardized regression coefficients.

\section{Results}

In this sample, $14.4 \%$ of participants scored $\geq 7$ on the SBQ-R, indicating clinically significant suicide risk compared to an adult general population, and $8.8 \%$ of participants scored $\geq 8$ on the SBQ-R, indicating clinically significant suicide risk compared to an adult psychiatric inpatient population. To examine the extent to which all of the study variables were correlated, Pearson's $r$ correlations were used. These values are illustrated in Table 2.

As illustrated in Figure 1, the indirect effect of PCa-QoL on suicide risk through both depression and psychache (PCa-QoL $\rightarrow$ Depression $\rightarrow$ Psychache $\rightarrow$ Suicide Risk) was significant, $b=-0.016$, CI [-0.027, -0.006]. In other words, depression and psychache, in serial, mediated the relationship between $\mathrm{PCa}-\mathrm{QoL}$ and suicide risk. Poorer PCa-QoL was related to greater levels of depressive symptoms, $b=-0.159, t(382)=-21.612, p<.001$, which in turn were related to greater psychache, $b=0.969, t(381)=11.006, p<.001$, which were associated with greater suicide risk, $b=0.103, t(379)=5.195, p<.001$. As hypothesized, the indirect effect of $\mathrm{PCa}-\mathrm{QoL}$ on suicide risk through depression and $\mathrm{PB} / \mathrm{TB}$ as serial mediators $(\mathrm{PCa}-\mathrm{QoL} \rightarrow$ Depression $\rightarrow \mathrm{PB} / \mathrm{TB} \rightarrow$ Suicide Risk) was significant, $b=-0.005$, CI $[-0.011,-0.001]$. Poorer $\mathrm{PCa}-\mathrm{QoL}$ was related to greater levels of depressive symptoms (same effect as above), which then were associated with greater $\mathrm{PB} / \mathrm{TB}, b=0.497, t(381)=4.029, p<.001$, which in turn were related to greater suicide risk, $b=0.064, t(379)=4.534, p<.001$. Of note, the magnitude of the mediation effect of depression in serial with psychache was significantly stronger than that of depression in serial with PB/TB (CI $[-0.022,-0.001]$ ), contrary to the hypothesis.

Several of the lower order mediation effects were also significant. The indirect effect of PCa-QoL on suicide risk through psychache alone (PCa-QoL $\rightarrow$ Psychache $\rightarrow$ Suicide Risk) was 
significant, $b=-0.006$, CI $[-0.014,-0.001]$; poorer PCa-QoL was significantly associated with greater psychache, $b=-0.062, t(381)=-3.288, p=.001$, and greater psychache was significantly related to greater suicide risk (same effect as above). The indirect effect of $\mathrm{PCa}-$ QoL on suicide risk through $\mathrm{PB} / \mathrm{TB}$ alone ( $\mathrm{PCa}-\mathrm{QoL} \rightarrow \mathrm{PB} / \mathrm{TB} \rightarrow$ Suicide Risk) was also significant, $b=-0.012$, CI $[-0.019,-0.005]$; poorer PCa-QoL was significantly related to greater $\mathrm{PB} / \mathrm{TB}, b=-0.178, t(381)=-6.755, p<.001$, which in turn was associated with greater suicide risk (same effect as above). Notably, the magnitude of the mediation effects of psychache and $\mathrm{PB} / \mathrm{TB}$ did not significantly differ (CI $[-0.006,0.014])$. The indirect effect of PCa-QoL on suicide risk through depression alone ( $\mathrm{PCa}-\mathrm{QoL} \rightarrow$ Depression $\rightarrow$ Suicide Risk) was not significant, $b=-0.007, \mathrm{CI}[-0.025,0.008]$; although poorer PCa-QoL was significantly related to greater levels of depressive symptoms (same as above), greater levels of depressive symptoms were not significantly related to suicide risk, $b=0.044, t(379)=1.181, p=.238$. The direct effect of PCa-QoL on suicide risk was significant, $b=0.020, t(379)=2.759, p=.006$. In a follow-up subscale analysis, the relative influence of $\mathrm{PB} / \mathrm{TB}$ as separate mediators in the relationship between $\mathrm{PCa}-\mathrm{QoL}$ and suicide risk through depression was examined. Both $\mathrm{PB}$ and TB in serial with depression were significant mediators in the relationship between PCa-QoL and suicide risk. The magnitude of each of the serial mediation effects did not significantly differ. See Table 3 for a summary of the mediation model effects.

\section{Discussion}

This study examined suicidal behaviour among men with PCa with the aim of estimating the proportion of $\mathrm{PCa}$ patients at risk for suicide and outlining psychosocial predictors of suicide risk informed by two prominent theories of suicide. Compared to a general adult population, $14.4 \%$ of individuals in the current sample were clinically at-risk for suicide, an estimate similar to that in other chronic pain and chronic disease samples. ${ }^{35}$

The results were generally in line with the hypotheses. Depression, in serial with the theory variables of psychache and the combination of $\mathrm{PB}$ and $\mathrm{TB}$, significantly mediated the effect of $\mathrm{PCa}-\mathrm{QoL}$ on suicide risk. Thus, worse $\mathrm{PCa}-\mathrm{QoL}$ was related to higher depression, which in turn related to both greater psychache and $\mathrm{PB} / \mathrm{TB}$, which promoted greater suicide risk. Interestingly, psychache stood out as a significantly stronger mediator in comparison with $\mathrm{PB} / \mathrm{TB}$, suggesting that psychache may levy more effect on suicide risk in this sample. This finding closely aligns with the view that psychache is a leading variable associated with suicidal behaviour. ${ }^{15,19}$ Moreover, the finding that depression was not a significant mediator between QoL and suicide risk after accounting for the influence of the two theory variables provides further support for the notion posited in the psychache theory that all other predictors (e.g., depression) are relevant to suicidal behaviour only in terms of their association with psychache. ${ }^{15}$ However, the finding that the serial mediation effect of depression and psychache is significantly stronger than that of depression and PT/TB should be considered with caution as it reflects a statistical difference, which is not to suggest that interpersonal factors should completely be discounted as treatment targets in PCa populations. To better understand the influence of the 
IPTS on suicide risk, the INQ-10 was broken down into the PB and TB subscales. PB and TB were compared against one another in a mediation model to determine the relative roles of these variables in the relationship between $\mathrm{PCa}-\mathrm{QoL}$ and suicide risk. Both subscales were significant mediators with neither being significantly stronger than the other, suggesting that both aspects of the IPTS are relevant in mediating the PCa-QoL suicide risk relationship in this population.

Based on these findings, psychological pain and perceived social fit should be focused on within PCa management. Psychological therapies, such as cognitive behavioral therapy, are successful treatments for those suffering from major depressive disorders ${ }^{36}$ and have positive effects in cancer patients. ${ }^{37}$ As well, simple interventions such as exercise and peer support, aid in the treatment of depression in some PCa patients. ${ }^{38}$ The current study findings highlight the importance of both intra- and interpersonal factors in the development of suicide risk. As such, a critical next step in understanding how best to provide care to PCa patients would be to examine the difference between individual and group-based interventions.

A primary study limitation is the homogenous nature of the sample, as approximately $98 \%$ of the participants were Caucasian, and over $80 \%$ were at least college-educated. This homogeneity limits the generalizability of the findings to other ethnic/cultural groups, which is of primary concern given that Black men are more likely to be diagnosed with PCa and nearly 2.5 times more likely to die of the disease compared to non-Hispanic white men. ${ }^{39}$ Data on clinical tumor characteristics and detailed treatment information (beyond what is presented in Table 1) is also lacking, but is partly ameliorated by the inclusion of PCa QoL information. Further, different types of PCa treatment may influence comorbidity development in PCa. PCa patients treated with androgen deprivation therapy have been reported to have a higher likelihood of developing new comorbidities. ${ }^{40}$ Given this limitation, we cannot conclude that the associations between the study variables apply to all PCa presentations. Future research should consider these biologic factors in their study design to examine their role in clinical outcomes. Another limitation to generalizability is self-selection bias associated with being a member of PCa support groups ${ }^{41}$ and participating in online studies. ${ }^{42}$ Online support groups are suggested to be important avenues for information and emotional support for PCa patients; however, individuals who participate in online PCa support groups tend to be younger, more highly educated, and earn a higher income compared to individuals who participate in in-person support groups $^{41}$. Consequently, the present sample may limit generalizability. The use of self-report measures and the cross-sectional nature of the study design may also be considered a limitation. However, given the focus of this study on providing initial support for a model of suicide risk in $\mathrm{PCa}$, future studies may be crafted to employ an interview style of data acquisition across multiple time points to track prospective changes in risk and the factors that predict it. Further, cross-sectional examinations of mediation models are considered valid. ${ }^{34} \mathrm{~A}$ final concern is that several variables likely important to PCa suicide risk, such as social support, remain unaccounted for in this study, which future research should consider. It is important to note that although this study models a linear arrangement of variables, these variables may be experienced by patients 
in a bidirectional manner. Considering this possibility may allow clinicians some extra measure of confidence that whatever area of this model is intervened upon will likely be helpful.

\section{Conclusions}

This study's results are the first to provide initial evidence that psychache and interpersonal factors (i.e., PB and TB) may act as mechanisms that help connect QoL and depressive symptoms to suicide risk in a PCa sample. These results uniquely build on the urologic oncology literature, emphasizing the importance of psychosocial factors in clinical outcomes, and the role that they may play in disease management. Given the high prevalence of suicidal behaviour measured in this population, clinicians should routinely query this symptom domain and refer accordingly. 


\section{References}

1. Siegel RL, Miller KD, Jemal A. Cancer statistics, 2019. CA Cancer J Clin 2019;69:7-34.

2. Canadian Cancer Statistics Advisory Committee. Canadian Cancer Statistics 2019. 2019. Available electronically at: https://www.cancer.ca/ /media/cancer.ca/CW/cancer\%20information/cancer\%20101/Can adian\%20cancer\%20statistics/Canadian-Cancer-Statistics-2019-EN.pdf?la=en. Accessed October 1, 2019.

3. American Cancer Society. Cancer Facts \& Figures 2019. 2019. Available electronically at: https://www.cancer.org/content/dam/cancer-org/research/cancer-facts-andstatistics/annual-cancer-facts-and-figures/2019/cancer-facts-and-figures-2019.pdf. Accessed October 1, 2019.

4. Miller KD, Siegel RL, Lin CC, et al. Cancer Treatment and Survivorship Statistics, 2016. CA Cancer J Clin 2016;66:271-289.

5. Steginga SK, Occhipinti S, Gardiner RF, et al. Prospective study of men's psychological and decision-related adjustment after treatment for localized prostate cancer. Urology 2004;63:751-756.

6. Watts S, Leydon G, Birch B, et al. Depression and anxiety in prostate cancer: A systematic review and meta-analysis of prevalence rates. BMJ Open 2014;4:e003901.

7. Ribeiro JD, Huang X, Fox KR, et al. Depression and hopelessness as risk factors for suicide ideation, attempts and death: Meta-analysis of longitudinal studies. $\mathrm{Br} \mathrm{J}$ Psychiatry 2018;212:279-286.

8. Bill-Axelson A, Garmo H, Lambe M, et al. Suicide risk in men with prostate-specific antigen-detected early prostate cancer: A nationwide population-based cohort study from PCABaSe Sweden. Eur Urol 2010;57:390-5.

9. Dalela D, Krishna N, Okwara J, et al. Suicide and accidental deaths among patients with non-metastatic prostate cancer. BJU Int 2016;118:286-97.

10. Fall K, Fang F, Mucci LA, et al. Immediate risk for cardiovascular events and suicide following a prostate cancer diagnosis: Prospective cohort study. PLoS Med 2009;6:e1000197.

11. Fang F, Keating NL, Mucci LA, et al. Immediate risk of suicide and cardiovascular death after a prostate cancer diagnosis: Cohort study in the United States. J Natl Cancer Inst 2010;102:307-14.

12. Klaassen Z, Jen RP, DiBianco JM, et al. Factors associated with suicide in patients with genitourinary malignancies. Cancer 2015;121:1864-1872.

13. Smith DP, Calopedos R, Bang A, et al. Increased risk of suicide in New South Wales men with prostate cancer: Analysis of linked population-wide data. PLoS One 2018;13: e0198679.

14. Llorente MD, Burke M, Gregory GR, et al. Prostate cancer: A significant risk factor for late-life suicide. Am J Geriatr Psychiatry 2005;13:195-201.

15. Shneidman ES. Suicide as psychache: A clinical approach to self-destructive behavior. Rowman \& Littlefield Publishers, 1993.

16. Pompili M, Lester D, Leenaars AA, et al. Psychache and suicide: A preliminary investigation. Suicide Life Threat Behav 2008;38:116-121. 
17. Troister T, Davis MP, Lowndes A, et al. A five-month longitudinal study of psychache and suicide ideation: Replication in general and high-risk university students. Suicide Life Threat Behav 2013;43:611-20.

18. Troister T, Holden RR. A two-year prospective study of psychache and its relationship to suicidality among high-risk undergraduates. J Clin Psychol 2012;68:1019-27.

19. Verrocchio MC, Carrozzino D, Marchetti D, et al. Mental pain and suicide: A systematic review of the literature. Front Psychiatry 2016;7:1-14.

20. Van Orden KA, Witte TK, Cukrowicz KC, et al. The interpersonal theory of suicide. Psychol Rev 2010;117:575-600.

21. Chu C, Buchman-Schmitt JM, Stanley IH, et al. The interpersonal theory of suicide: A systematic review and meta-analysis of a decade of cross-national research. Psychol Bull 2017; 143:1313-45.

22. Ma J, Batterham PJ, Calear AL, et al. A systematic review of the predictions of the interpersonal-psychology theory of suicidal behavior. Clin Psychol Rev 2016;46:34-45.

23. Fervaha G, Izard JP, Tripp DA, et al. Depression and prostate cancer: A focused review for the clinician. Urol Oncol 2019;37:282-88.

24. Mohamed NE, Bovbjerg DH, Montgomery GH, et al. Pretreatment depressive symptoms and treatment modality predict post-treatment disease-specific quality of life among patients with localized prostate cancer. Urol Oncol 2012;30:804-12.

25. Izard J, Siemens DR, Verreault P, et al. MP21-19 The prevalence and biopsychosocial predictors of suicidality in men with prostate cancer. J Urol 2018;199:e270.

26. Gibson S, Boden ZV, Benson O, et al. The impact of participating in suicide research online. Suicide Life Threat Behav 2014;44:372-83.

27. Esper P, Mo F, Chodak G, et al. Measuring quality of life in men with prostate cancer using the functional assessment of cancer therapy-prostate instrument. Urology 1997;50:920-28.

28. Webster K, Odom L, Peterman A, et al. The Functional Assessment of Chronic Illness Therapy (FACIT) measurement system: Validation of version 4 of the core questionnaire. Qual Life Res 1999;604.

29. Kroenke K, Spitzer RL, Williams JB. The PHQ-9: Validity of a brief depression severity measure. J Gen Intern Med 2001;16:606-13.

30. Holden RR, Mehta K, Cunningham EJ, et al. Development and preliminary validation of a scale of psychache. Can J Behav Sci 2001;33:224-32.

31. Van Orden KA, Cukrowicz KC, Witte TK, et al. Thwarted belongingness and perceived burdensomeness: Construct validity and psychometric properties of the Interpersonal Needs Questionnaire. Psychol Assess 2012;24:197-215.

32. Osman A, Bagge CL, Gutierrez PM, et al. The Suicidal Behaviors Questionnaire-Revised (SBQ-R): Validation with clinical and nonclinical samples. Assessment 2001;8:443-54.

33. Tabachnick BG, Fidell LS. Using multivariate statistics, $5^{\text {th }}$ edition. Allyn \& Bacon/Pearson Education, 2007.

34. Hayes AF. Introduction to mediation, moderation, and conditional process analysis: A regression-based approach $2^{\text {nd }}$ edition. Guilford Press, 2018.

35. Calandre EP, Rico-Villademoros F, Slim M. Suicidal behaviors in patients with rheumatic diseases: A narrative review. Rheumatol Int 2018;38:537-48. 
36. Qaseem A, Barry MJ, Kansagara D. Nonpharmacologic versus pharmacologic treatment of adult patients with major depressive disorder: A clinical practice guideline from the American College of Physicians. Ann Intern Med 2016;164:350-9.

37. Van de Wal M, Thewes B, Gielissen M, et al. Efficacy of blended cognitive behavior therapy for high fear of recurrence in breast, prostate, and colorectal cancer survivors: The SWORD study, a randomized controlled trial. J Clin Oncol 2017;35:2173-83.

38. Newby TA, Graff JN, Ganzini LK, et al. Interventions that may reduce depressive symptoms among prostate cancer patients: A systematic review and meta-analysis. Psychooncology 2015;24:1686-93.

39. DeSantis CE, Siegel RL, Sauer AG, et al. Cancer statistics for African Americans, 2016: Progress and opportunities in reducing racial disparities. CA Cancer J Clin 2016;66:290308.

40. Ng HS, Koczwara B, Roder D, et al. Development of comorbidities in men with prostate cancer treated with androgen deprivation therapy: An Australian population-based cohort study. Prostate Cancer Prostatic Dis 2018;21:403-10.

41. Huber J, Thomas C, Karschuck P, et al. Online support groups for patients with prostate cancer. Aktuel Urol 2019;50:392-7.

42. Keiding N, Louis TA. Web-based enrollment and other types of self-selection in surveys and studies: Consequences for generalizability. Annu Rev Stat Appl 2018;5:25-47.

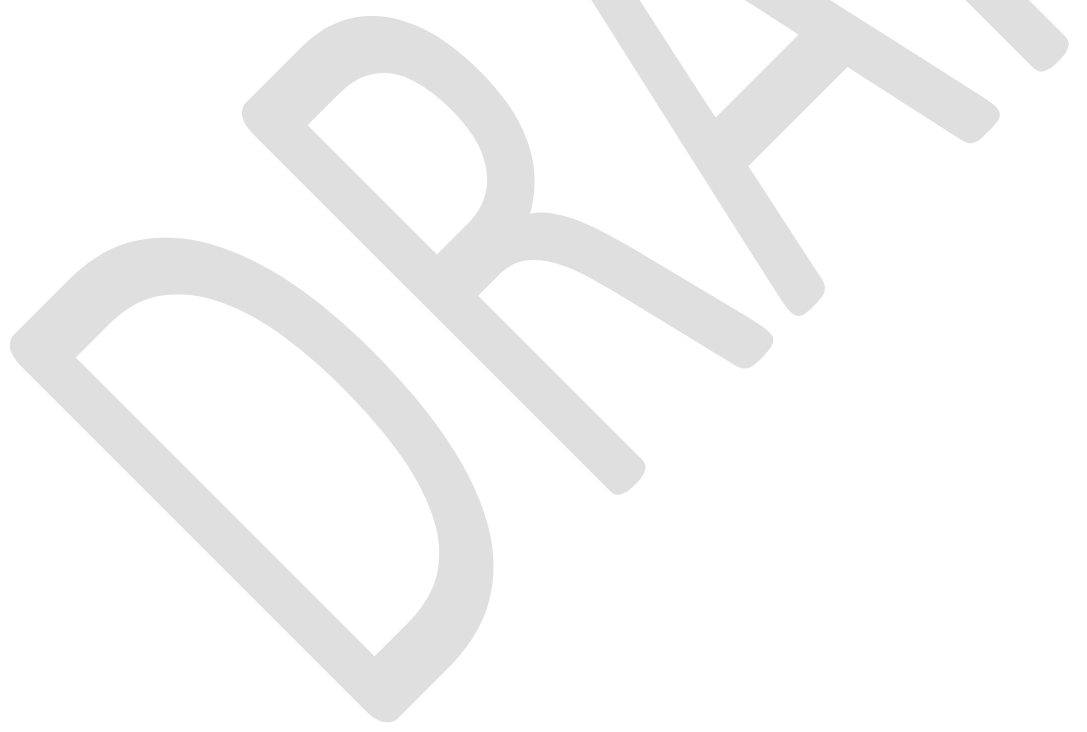




\section{Figures and Tables}

Fig. 1. Model of the mediation effects of depression in serial with body psychache and perceived burdensomeness/thwarted belongingness (PB/TB) (operating in parallel) in the relationship between prostate cancer quality of life (PCa-QoL) and suicide risk. Note that solid lines represent significant paths and dashed lines represet non-significant paths.

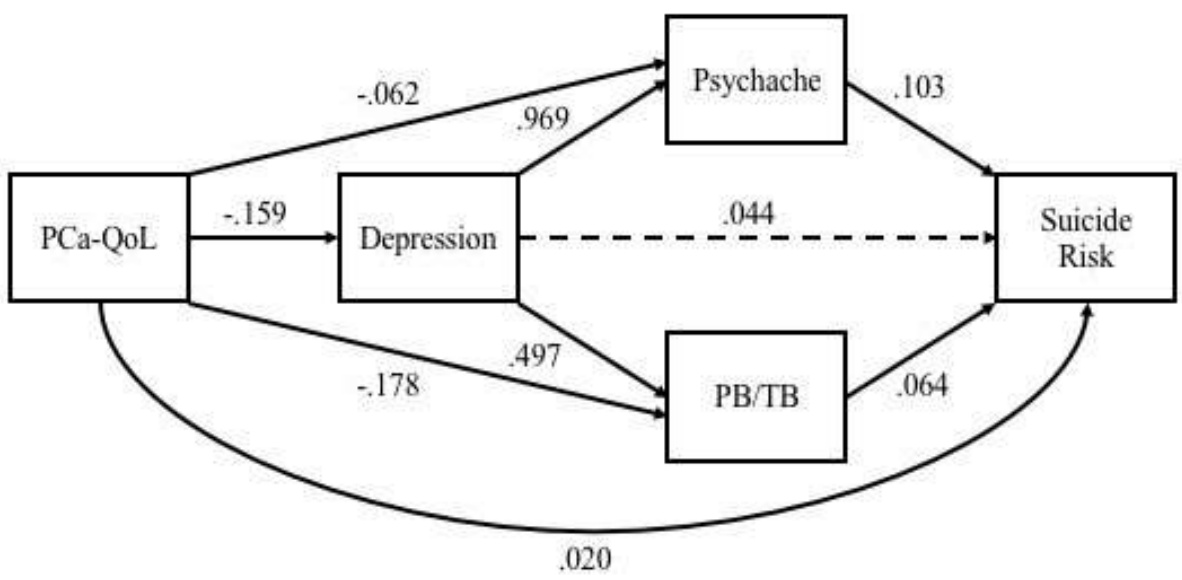

\begin{tabular}{|l|c|}
\hline Table 1. Demographic information for participants \\
\hline Age $(\mathrm{n}=398)$ & $\mathrm{M}=69.35, \mathrm{SD}=7.797$ \\
\hline Ethnicity $(\mathrm{n}=405)$ & $\mathbf{n}(\mathbf{\%})$ \\
\hline Caucasian/White & $396(97.8 \%)$ \\
Hispanic or Latino & $1(0.2 \%)$ \\
Aboriginal & $1(0.2 \%)$ \\
Asian & $3(0.7 \%)$ \\
Black/African-Canadian & $3(0.7 \%)$ \\
Other & $1(0.2 \%)$ \\
\hline Continent of residence (n=404) & $\mathbf{n}(\mathbf{\%})$ \\
\hline Europe & $49(12.1 \%)$ \\
North America & $257(63.6 \%)$ \\
Australia and Oceania & $98(24.3 \%)$ \\
\hline Education level (n=405) & $\mathbf{n}(\mathbf{\%})$ \\
\hline Less than high school & $11(2.7 \%)$ \\
High school or GED & $59(14.6 \%)$ \\
Some college/university & $74(18.3 \%)$ \\
Graduate from college/university & $143(35.3 \%)$ \\
Graduate or professional school after college/university & $118(29.1 \%)$ \\
\hline
\end{tabular}




\begin{tabular}{|c|c|}
\hline Living with spouse/partner $(\mathrm{n}=371)$ & $\mathbf{n}(\mathbf{\%})$ \\
\hline Yes & $347(93.5 \%)$ \\
No & $24(6.5 \%)$ \\
\hline Employment status $(\mathrm{n}=405)$ & $\mathbf{n}(\mathbf{\%})$ \\
\hline Employed & $95(23.5 \%)$ \\
Unemployed & $8(2.0 \%)$ \\
Retired & $295(72.8 \%)$ \\
Disabled & $6(1.5 \%)$ \\
Student & $1(0.2 \%)$ \\
\hline Type of prostate cancer $(\mathrm{n}=400)$ & $\mathbf{n}(\mathbf{\%})$ \\
\hline Localized PCa & $315(78.8 \%)$ \\
Advanced or metastatic PCa & $85(21.3 \%)$ \\
\hline Length of diagnosis (n=405) & $\mathbf{M = 6 . 3 ~ y e a r s , ~ S D = 5 ~ y e a r s ~}$ \\
\hline Suicide risk (n=388) & $\mathbf{n}(\mathbf{\%})$ \\
\hline Participants at risk for suicide according to the general & $56(14.4 \%)$ \\
population cut-off (score $\geq 7)$ & $34(8.8 \%)$ \\
Participants at risk for suicide according to the in- & $\mathbf{n}(\mathbf{\%})$ \\
patient cutoff (score $\geq 8)$ & $162(36.7)$ \\
\hline Radiation therapy & $135(30.5 \%)$ \\
Hormone therapy (androgen deprivation therapy) & $21(4.8 \%)$ \\
Chemotherapy & $87(19.7 \%)$ \\
Other & \\
\hline Treatments received & \\
\hline
\end{tabular}

M: median; PCa: prostate cancer; SD: standard deviation.

Table 2. Correlation matrix for predictor variables and suicide risk

\begin{tabular}{|c|c|c|c|c|c|}
\hline & 1 & 2 & 3 & 4 & 5 \\
\hline PCa-QoL (1) & 1 & & & & \\
\hline Depression (2) & $-0.740^{* *}$ & 1 & & & \\
\hline $\mathrm{PB} / \mathrm{TB}(3)$ & $-0.592^{* *}$ & $0.536^{* *}$ & 1 & & \\
\hline Psychache (4) & $-0.608^{* *}$ & $0.712^{* *}$ & $0.603^{* *}$ & 1 & \\
\hline Suicide risk (5) & $-0.247^{* *}$ & $0.346^{* *}$ & $0.409^{* *}$ & $0.463^{* *}$ & 1 \\
\hline
\end{tabular}




\begin{tabular}{|l|c|}
\hline \multicolumn{2}{|l|}{ Table 3. Indirect and direct effects from mediation model } \\
\hline Path & Effect \\
\hline Serial mediation effects & $-0.016^{*}$ \\
\hline PCa-QoL $\rightarrow$ Depression $\rightarrow$ Psychache $\rightarrow$ Suicide Risk & $-0.005^{*}$ \\
\hline PCa-QoL $\rightarrow$ Depression $\rightarrow$ PB/TB $\rightarrow$ Suicide Risk & -0.007 \\
\hline Lower order mediation effects & $-0.006^{*}$ \\
\hline PCa-QoL $\rightarrow$ Depression $\rightarrow$ Suicide Risk & $-0.012^{*}$ \\
\hline PCa-QoL $\rightarrow$ Psychache $\rightarrow$ Suicide Risk & \\
\hline PCa-QoL $\rightarrow$ PB/TB $\rightarrow$ Suicide Risk & $0.020^{*}$ \\
\hline Direct effect
\end{tabular}

"Effect is significant (95\% confidence interval did not contain zero). PB/TB: perceived burdensomeness/thwarted belongingness; $\mathrm{PCa} / \mathrm{QoL}$ : prostate cancer quality of life. 